\title{
Fine needle aspiration cytology in the diagnosis of Tuberculous lymphadenitis and utility of Ziehl Neelsen stain benefits and pitfalls
}

\author{
Vimal $S^{1}$, Dharwadkar $A^{2}$, Chandanwale Shrish $S^{3}$, Verma $V^{4}$, Khandelwal $A^{5}$ \\ ${ }^{1}$ Dr Shruti Vimal, Associate Professor, Department of Pathology, DYPMC, Pimpri Pune, ${ }^{2}$ Dr Arpana Dharwadkar, \\ Associate Professor, Department of Pathology, DYPMC, Pimpri Pune ${ }^{3}$ Dr Shrish S Chandanwale, Professor, Department \\ of Pathology, DYPMC, Pimpri Pune, ${ }^{4}$ Dr Vandana Verma, Consultant, Private Hospital, Delhi, ${ }^{5}$ Dr Anushree \\ Khandelwal, Post graduate student, Department of Pathology, DYPMC, Pimpri Pune, Maharashtra, India.
}

Address for Correspondence: Dr Shruti Vimal, Email: shruti.vimal@gmail.com

\begin{abstract}
Introduction: In a developing country like India, tuberculous lymphadenitis is one of the most common presentations at OPDs. However, anti-tubercular treatment cannot be administered only on clinical suspicion. Cytomorphology with acid fast staining proves to be a valuable tool in diagnosing these cases along with culture. The study was undertaken to study the utility, limitations of fine needle aspiration cytology and various cytomorphological presentations in reference to Ziehl-Neelsen staining in tuberculous lymphadenitis and correlate the culture findings. Methods: The study was conducted for duration of two years with total of 170 cases at a tertiary care centre. The patients with clinically suspected lymphadenopathy were selected. Results: The incidence of tuberculous lymphadenitis was 68.8\%. Overall AFB positivity was $64.1 \%$. Epithelioid cell granulomas with lymphocytes were the most common cytological picture and cases showing necrosis had highest AFB positivity. Maximum patients presented in second to fourth decade of life. Cervical region was the most common site of involvement with solitary lymphadenopathy as the most common presentation in contrast to matted lymph nodes as reported by others. Conclusion: Yet again Fine needle aspiration cytology is a safe, cheap and reliable procedure requiring minimal instrumentation and is highly sensitive to diagnose tuberculous lymphadenitis. The diagnostic index can be further increased by complementing cytomorphology with acid fast staining and culture techniques. However FNAC complimented with techniques like ELISA and PCR would give better dimensions to the current scenario of diagnosis and treatment modalities.
\end{abstract}

Key words: Lymphadenitis, FNAC, Tubercular Lymphadenitis, Granulomatous, AFB.

\section{Introduction}

Ancient man described diseases to be work of an evil spirit, curse brought on man by the wrath of malevolent Gods, or the act of supernatural powers. With evolvement of medical sciences, pioneers Louis Pasteur and Robert Koch shared the insight that diseases were caused by bacteria which evoked specific response in the body. Granulomatous response is one such response. Granulomatous lymphadenitis is a manifestation of several conditions including mycotic, viral and bacterial infections like tuberculosis, leprosy, syphilis, Sarcoidosis, toxoplasmosis and secondary response in lymph nodes draining carcinomas or lymphomas [1]. Tuberculosis is a major health hazard in developing

Manuscript received $14^{\text {th }}$ July 2016

Reviewed: $25^{\text {th }}$ July 2016

Author Corrected: $6^{\text {th }}$ August 2016

Accepted for Publication $20^{\text {th }}$ August 2016 countries. In India 1000 people per day or one per min account for mortality due to tuberculosis [2,3,4]. Extra pulmonary tuberculosis is on rise world over. It's a protean disease, which can virtually affect all organs [5]. The global increase is believed to be fuelled by HIV related immunosuppression. Peripheral lymph node involvement is the commonest form of extra pulmonary mycobacterial disease and cervical region is the most frequently affected [3,6,7]. Fine Needle Aspiration Cytology (FNAC) provides an inexpensive, quick and safe alternative to histopathology for the diagnosis of tuberculosis. It's a patient friendly technique and provides a good assessment of cytomorphological features. The presence of epithelioid granuloma forms the basis of diagnosis of tuberculosis. Demonstration of AFB in FNAC smears directly or by culture finally nails 
the etiology, even if epithelioid cell granulomas are not seen $[2,8]$. The present study was undertaken to evaluate the diagnostic value of FNAC in Tuberculous lymphadenitis and the utility of Ziehl-Neelsen staining, to find the prevalence of tuberculosis in clinically suspected patients of tuberculous lymphadenitis and to analyze the cytomorphological features encountered in aspirates of tuberculous lymphadenitis and correlate them with acid fast bacilli positivity on Ziehl Neelsen stain and with the results of culture.

\section{Materials and Method}

The work represents the prospective study undertaken in the department of pathology at a tertiary care centre over a period of 2 yrs. Patient selection was clinically suspected tuberculous lymphadenitis irrespective of age, sex and site of lymphadenopathy. A detailed history of the patient was taken and a through clinical examination was performed.

\section{Results}

All the patients of lymphadenopathy with a clinical suspicion of tuberculosis were included in the study. A total of 170 cases have been studied out of which 117 were diagnosed as tubercular lymphadenitis, 41 reactive lesions, 3 metastatic deposits, single case of NHL, two cystic lesions and six cases were inconclusive. (Table 1)

\section{Table-1: Relative frequency of Lymph node lesions.}

\begin{tabular}{|c|c|c|}
\hline Lymph Node Lesions & No. & Percentage \\
\hline Tuberculous lymphadenitis & 117 & 68.8 \\
\hline Reactive lymphadenitis & 41 & 1.7 \\
\hline Metastatic deposits & 3 & 0.5 \\
\hline Non Hodgkin's Lymphoma & 1 & 1.1 \\
\hline Benign Cystic Disease & 2 & 3.5 \\
\hline Inconclusive Aspirates & 6 & \\
\hline
\end{tabular}

Amongst the cases of tuberculous lymphadenitis, 46 were male and 71 were females making M: F ratio 1:1.54 and a slight predominance were noted in second to fourth decades of life. Associated clinical features like low grade fever, anorexia, malaise and weight loss were present in 78 patients (66.6\%). Ninety seven patients (82.9\%) had lymphadenopathy of more than one week. Cervical lymphadenopathy was seen in $66.6 \%$, followed by supraclavicular in $23 \%$, axillary nodes in 16 cases and inguinal in 4.2\%. (Table 2) Amongst the cervical lymph node 39 lymph node enlargements were noted in the head and around the external jugular vein. Single enlarged lymph nodes were noted in $58.9 \%$ of cases, followed by $27.3 \%$ showing multiple and discrete lymph nodes and matted lymph nodes were seen in total of $13.6 \%$ of cases.

Table-2: Site of Lymph Nodes.

\begin{tabular}{|c|c|}
\hline Site of lymphadenopathy & Percentage of cases \\
\hline Superficial cervical lymph nodes & $38 \%$ \\
\hline Supraclavicular lymph nodes & $26 \%$ \\
\hline Axillary lymph nodes & $15 \%$ \\
\hline Inguinal lymph nodes & $5 \%$ \\
\hline Generalized Lymphadenopathy & $16 \%$ \\
\hline
\end{tabular}


The lymph nodes varied in size ranging from less than $1 \mathrm{~cm}$ to more than $2 \mathrm{~cm}$. Majority of cases were recorded in the criterion of less than $1 \mathrm{~cm}(38.4 \%)$. A firm consistency of lymph node was observed in 79 cases $(67.5 \%)$, while 38 cases $(32.4 \%)$ were soft. The aspirate was admixed with blood in 58 cases $(48.7 \%)$, while it was cheesy in $23(19.6 \%)$ and purulent in 36 cases $(30.7 \%)$.

In the current study, following cytomorphological features were observed and the cases of tuberculous lymphadenitis were grouped as follows:

- Group I : Epithelioid cell granulomas without necrosis

- Group II: Epithelioid cell granulomas with necrosis.

- Group III: Only necrosis

- Group IV: Necrosis with poly morphonuclear leukocytosis.

The incidence of Group I was the highest among all being 34.1\%, thirty nine cases were included in Group II (33.3\%), seven in Group III (5.9\%) and 31 in Group IV (26.4\%). ZN stain was performed in all the cases. The ZN positivity was $32.5 \%$ in Group I, $64.1 \%$ in Group II, $71.4 \%$ in Group III and $67.7 \%$ in Group IV. Overall ZN positivity was $54.7 \%$. (Table 3).

Table-3: Correlation of Cytomorphological features and ZN stain.

\begin{tabular}{|c|c|c|}
\hline Group & Total No of Cases & Total No. of ZN Positive Cases \\
\hline Group I & $40(34.1)$ & $13(32.5 \%)$ \\
\hline Group II & $39(33.3 \%)$ & $25(64.1 \%)$ \\
\hline Group III & $7(5.9 \%)$ & $5(71.4 \%)$ \\
\hline Group IV & $31(26.4 \%)$ & $21(67.7 \%)$ \\
\hline
\end{tabular}

The ZN positivity was also compared with the nature of aspirate as $44.8 \%$ in material mixed with blood, $69.5 \%$ in cheesy aspirate and $61.1 \%$ in purulent material. Since the demonstration of AFB was diagnostic of tuberculosis, only the ZN negative cases were selected to be inoculated on LJ medium. Out of a total $53 \mathrm{ZN}$ negative cases, culture was attempted in 40 cases only, because of inadequacy of the aspirate and the patient being unwilling for a second attempt at FNAC. Amongst these 40 cases, culture was positive in 10 cases, hence making culture positivity $25 \%$.

Table-4: Culture Positivity.

\begin{tabular}{|c|c|c|c|}
\hline Groups & ZN Negative Cases & Culture Attempted & No. of Positive Cultures \\
\hline Group I & 27 & 20 & $10 \%$ \\
\hline Group II & 13 & 9 & $55.5 \%$ \\
\hline Group III & 2 & 2 & 0 \\
\hline Group IV & 11 & 9 & $33.3 \%$ \\
\hline
\end{tabular}

Among the 117 cases of tuberculous lymphadenitis, 16 were HIV seropositive. Regional lymph node involvement was seen in 12 cases and the other 4 showed generalized lymphadenopathy. Cervical nodes showed maximum presentation with a total of 8 cases, followed by axillary 3 cases and a single case was noted in inguinal region. A confirmative diagnosis of tubercular lymphadenitis was offered in 11 cases and the other five were $\mathrm{ZN}$ negative. Three amongst the latter showed epithelioid cell granulomas and the other two showed necrosis and poly morphonuclear inflammatory infiltrate and were inoculated for culture, amongst them one was culture positive.

Cytomorphologically, the most common category encountered was Group I \& IV, with 6 cases in each (37.5\%), followed by group II with 3 cases $(18.7 \%)$ and Group III with a single case $(6.2 \%)$. Maximum ZN positivity (100\%) was observed in Group II and Group III, followed by Group IV (66.6\%) and Group I (50\%). There were eleven ZN positive cases in total. (Table 5) 
Table- 5: Correlation of cytomorphological Features and ZN stain.

\begin{tabular}{|c|c|c|}
\hline Groups & No. of cases & ZN Positive Cases \\
\hline Group I & 6 & $50 \%$ \\
\hline Group II & 3 & $100 \%$ \\
\hline Group III & 1 & $100 \%$ \\
\hline Group IV & 6 & $66.6 \%$ \\
\hline
\end{tabular}

The ZN positivity was $100 \%$ in cheesy aspirates, followed by aspirates mixed with blood (66.6\%) and purulent aspirates (60\%). The overall ZN positivity was $68.7 \%$, maximum being in Group. II \& III and in cheesy aspirates.

The $5 \mathrm{ZN}$ negative cases were inoculated on LJ medium ( 3 cases in Group I and 2 cases in Group IV). The culture positivity was $66.6 \%$ in Group I ( 2 out of 3 cases were culture positive) and 50\% in group IV ( 1 out 2 cases was culture positive). The overall culture positivity was $60 \%$.

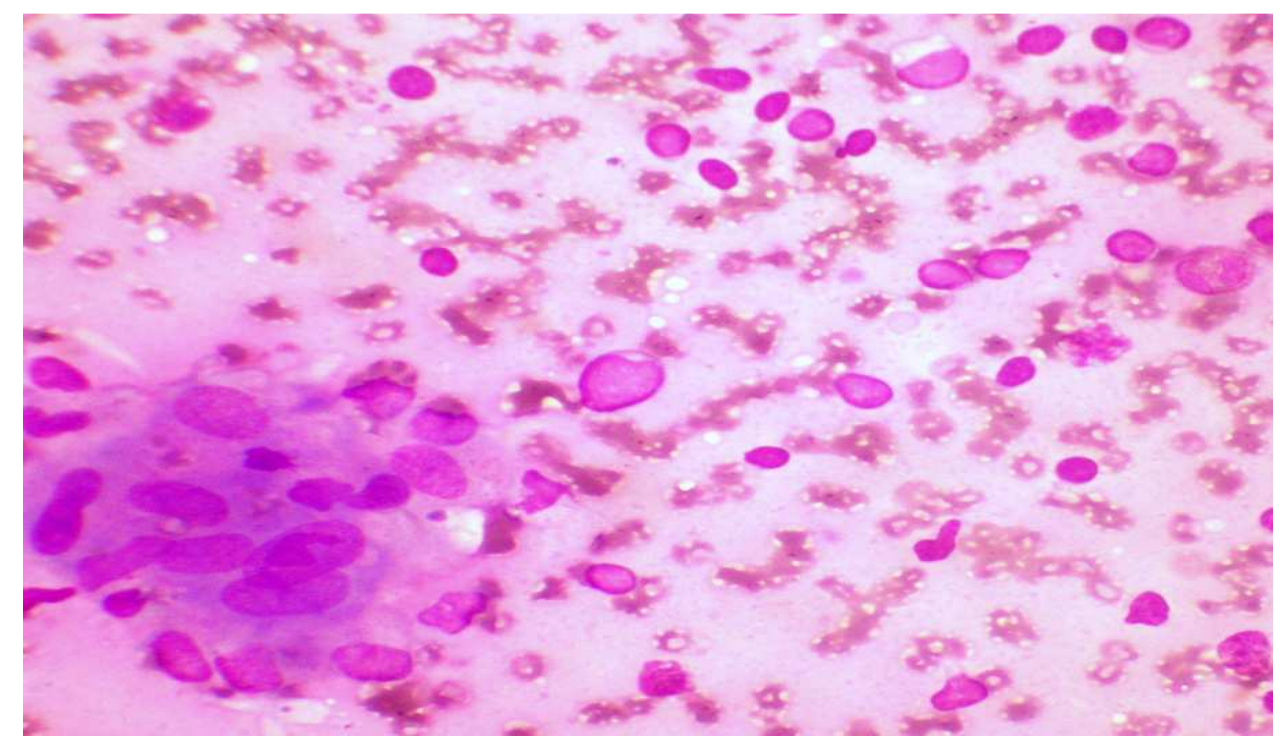

Figure-1: Epithelioid cell granuloma with few lymphocytes (Leishman 400x) Group I

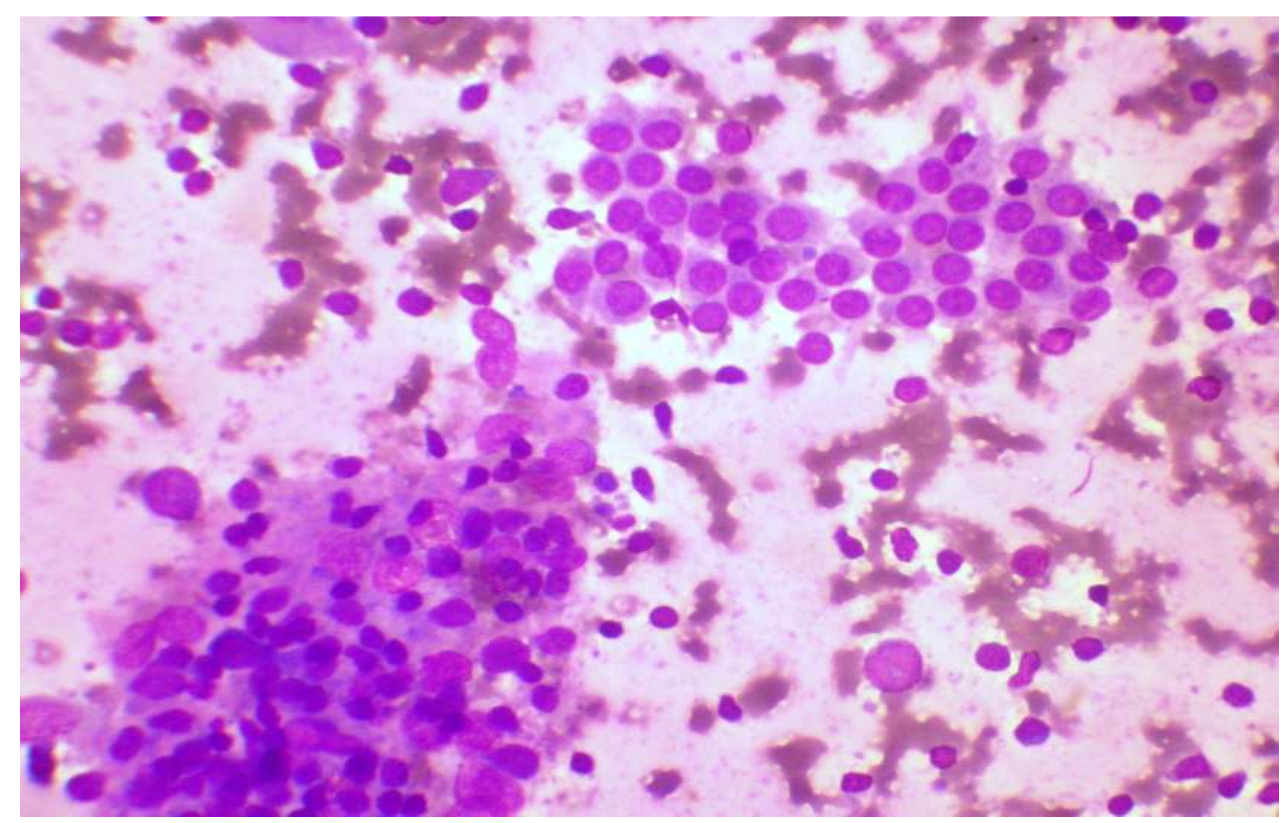

Figure-2: Epithelioid cells with necrosis and inflammatory cells (Leishman 400x) Group II 


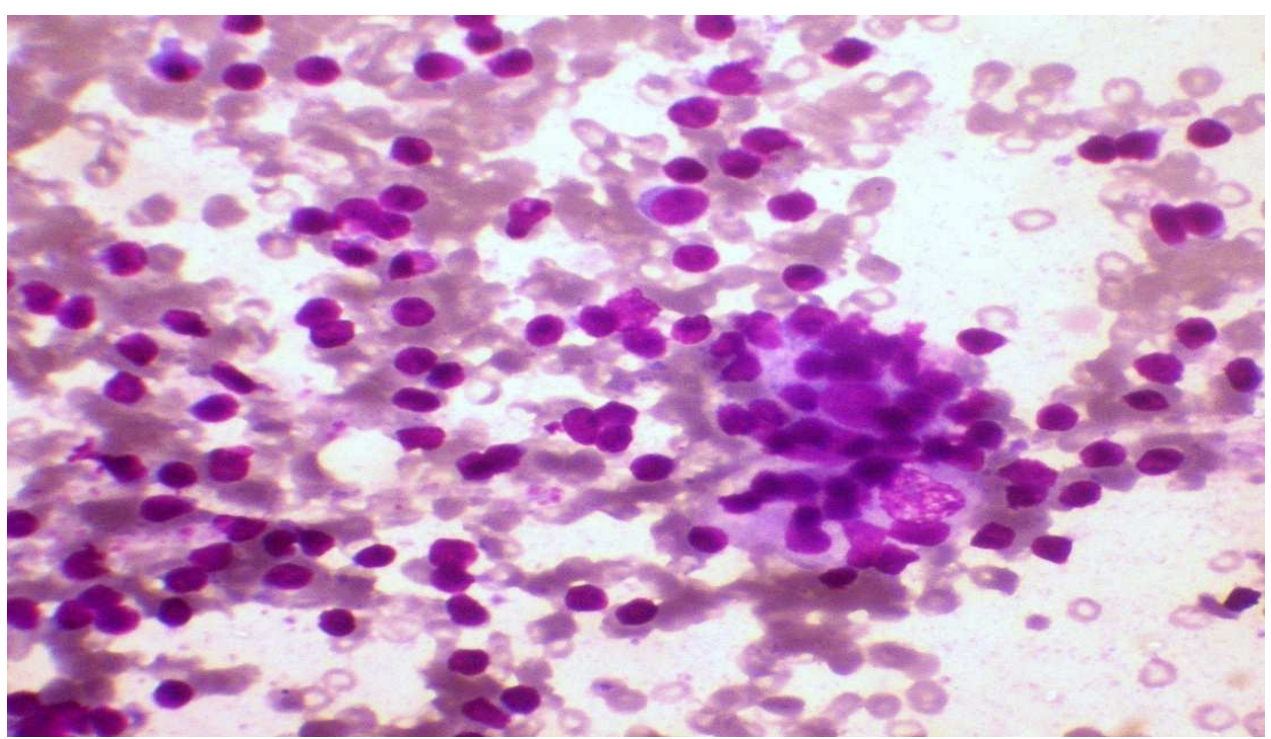

Figure-3: Epithelioid cells with necrosis and inflammatory cells (Leishman 400x) Group II

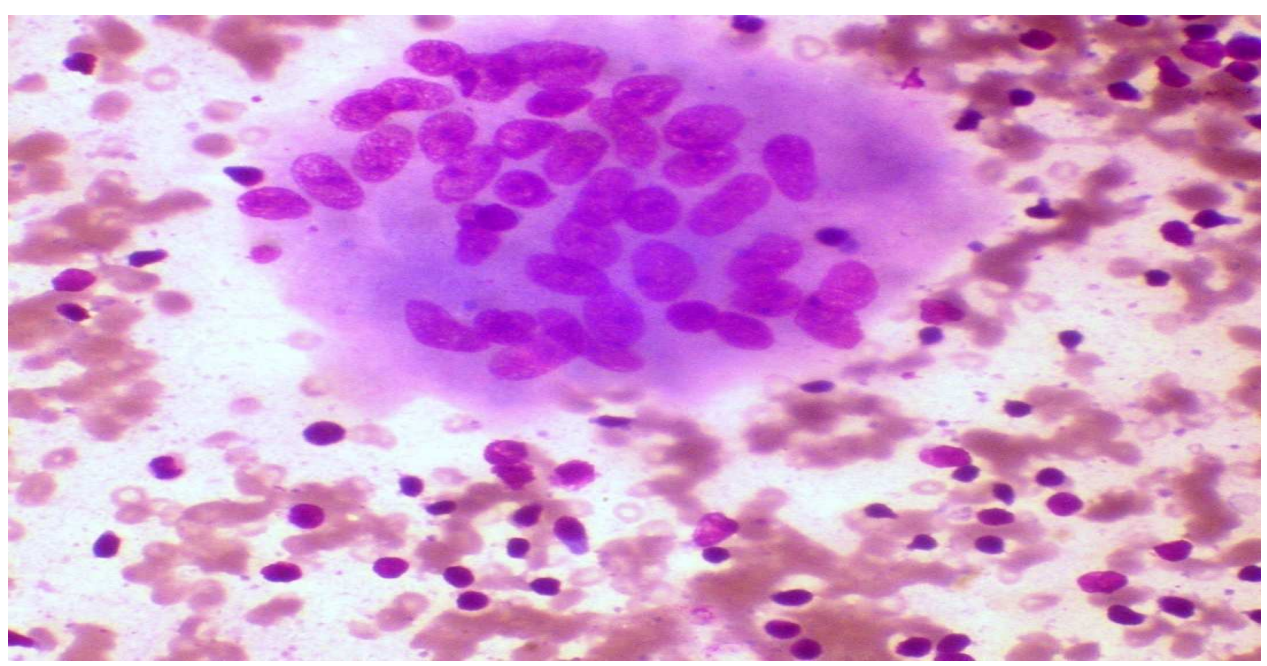

Figure- 4: Epithelioid cells with necrosis and mixed inflammatory cells polymorphs and lymphocytes (Leishman 400x) Group II

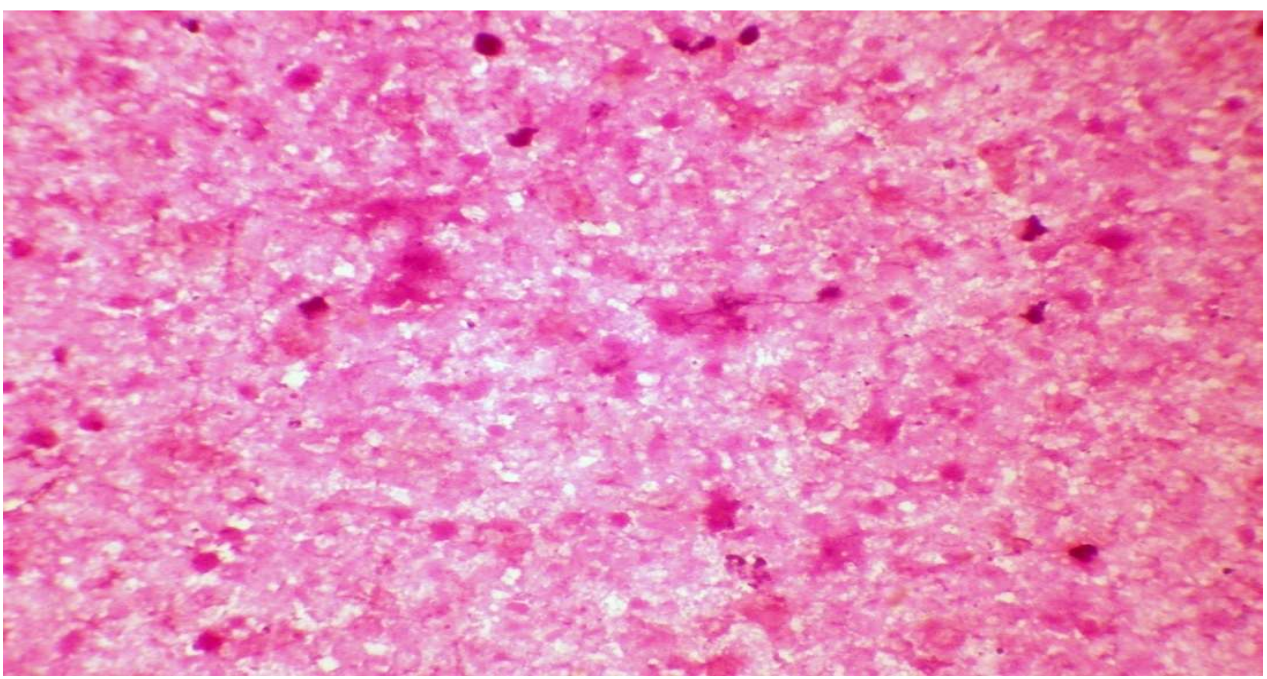

Figure-5: Necrosis (H\&E 400 x) Group III 


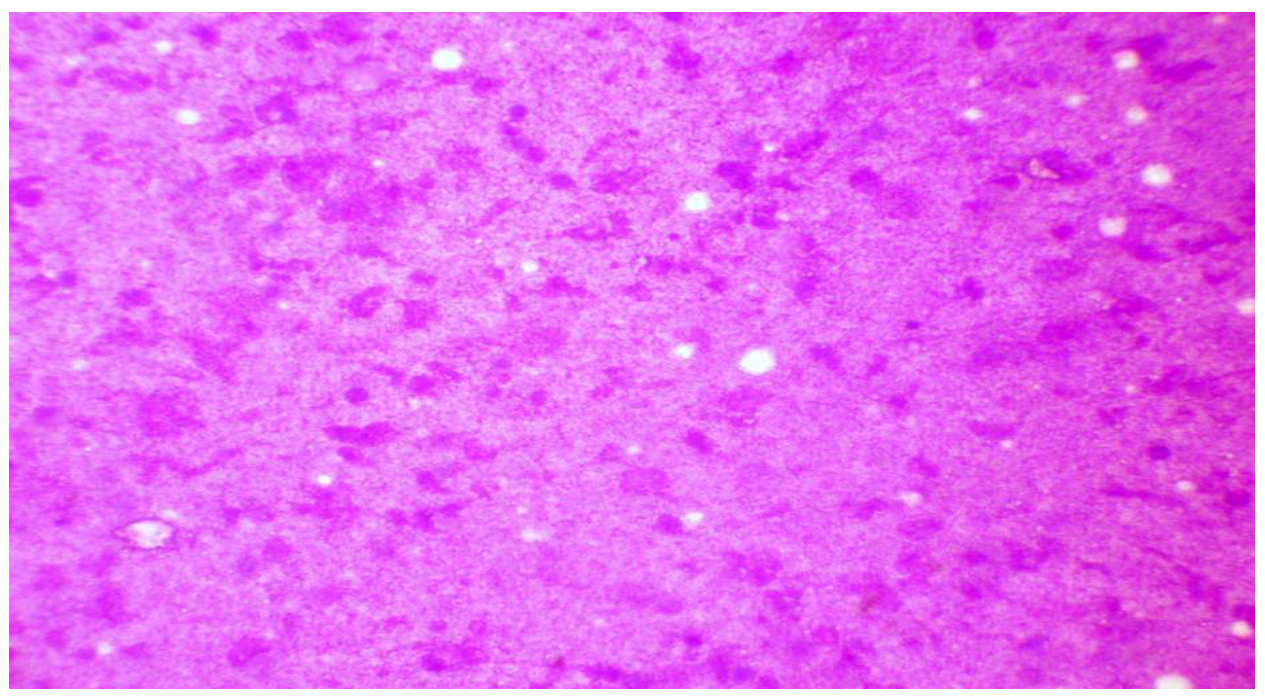

Figure-6: Necrosis (Leishman 400 x) Group III

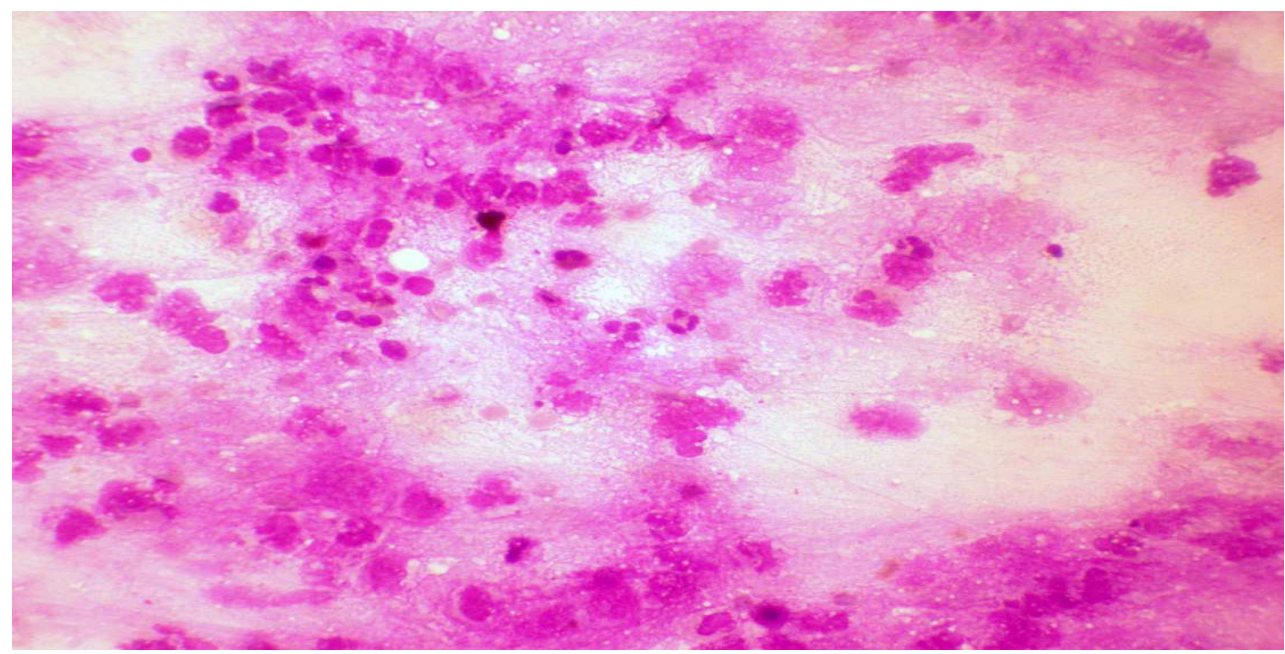

Figure-7: Necrosis and poly morpho nuclear leukocytes (Leishman 400x) Group IV

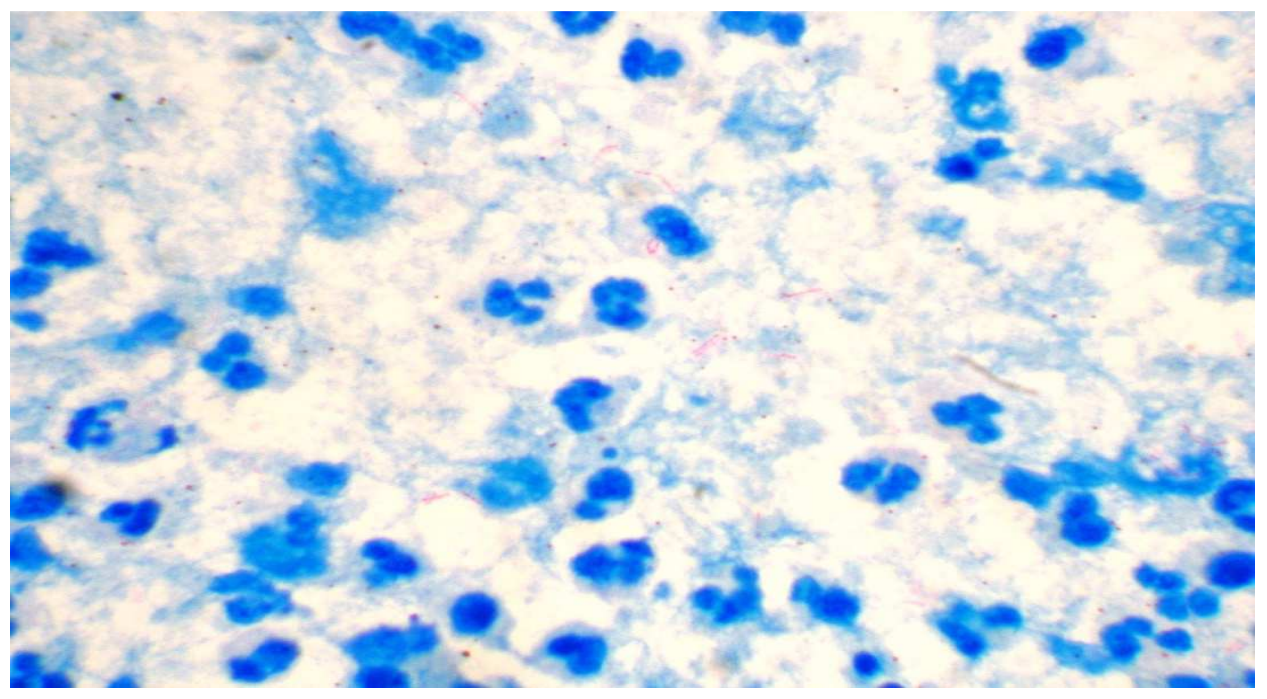

Figure-8: Acid Fast Bacilli (ZN Stain Oil immersion) 
Patients were treated with anti tubercular treatment. A clinical follow up of the patients receiving treatment from hospital was carried out. It was found that out of total 117 cases of tuberculous lymphadenitis 47 were taking anti tubercular treatment. Amongst these 28 were $\mathrm{ZN}$ positive and 19 were negative for $\mathrm{ZN}$. The material aspirated from 15 out of these 19 cases was inoculated on $\mathrm{LJ}$ media out of which 5 cases tested positive.

\section{Discussion}

Enlarged symptomatic lymphadenopathy most frequently involving head, neck, axilla and inguinal regions are relatively common clinical findings. The etiology ranges from simple reactive hyperplasia to tuberculosis and malignancies. Diagnosis of mycobacterial cervical lymphadenitis remains a diagnostic challenge for many clinicians despite current advances in diagnostic laboratory techniques. Fine needle aspiration is a simple, rapid and patient friendly diagnostic technique.

A total of 170 cases were aspirated out of which 117 $(68.8 \%)$ were diagnosed as tubercular lymphadenitis. More than half the patients $(56.4 \%)$ were in second and third decades and showed a female preponderance (M: F 1:1.5). These findings were in accordance with the studies conducted by Rajshekeran et al and Natraj et al. $[9,10]$.

Associated clinical features such as low grade fever, weight loss, anorexia and malaise were present in $66.6 \%$ cases. In the present series, majority of the patients $(58.9 \%)$ had single enlarged lymph node while in the study conducted by Seth and Donald multiple lymph node involvement was common. [11].

Considering the site superficial cervical lymph nodes were affected in most of the patients. Similar findings were observed by Kusum Verma et al [2], Rajshekaran et al [9], Das et al [12], Gupta et al [13] and Tripathy et al [14]. In contrast to above said findings Dandapat et al [7] and Madhusudan et al [15] observed upper deep cervical nodes to be more commonly involved.

The size of the lymph node ranged from $1 \mathrm{~cm}$ to $5 \mathrm{~cm}$ in diameter, with $38.4 \%$ being less than $1 \mathrm{~cm}$ and the rest were more than $1 \mathrm{~cm}$ in diameter $(61.5 \%)$. Bedi et al [16] and Ahmed et al [17] have suggested that smaller lymph nodes have less chances of being tuberculous and have experienced that lymph nodes up to $1 \mathrm{~cm}$ diameter are indicative of a reactive process. However in the present study lymph node $<1 \mathrm{~cm}$ was not accountable as a limiting factor.

A firm consistency was observed in $67.5 \%$ cases while the rest $(32.4 \%)$ were soft. Approximately half (48.7\%) yielded an aspirate mixed with blood, followed by $30.7 \%$ cases in which purulent material was obtained and in $19.6 \%$ cheesy material was aspirated. The above finding correlated with the clinical stage and immunological status of the patients.

Cytomorphologically the incidence of group I was highest $(34.1 \%)$, followed by Group II $(33.3 \%)$. There were $5.9 \%$ cases in group III and $26.4 \%$ in group IV. The $\mathrm{ZN}$ positivity ranged from $32.5 \%$ to $71.4 \%$ maximum being in Group III. This was followed closely by Group IV (67.7\%) and Group II (64.1\%). Group I showed the lowest ZN positivity $(32.5 \%)$. The overall $\mathrm{ZN}$ positivity was low $(54.7 \%)$ and that can be explained as its defined by the clinical stage and immunological status of the patient and a minimum of 5 to $10000 \mathrm{bacilli} / \mathrm{ml}$ of the aspirate are required to get a positive result on $\mathrm{ZN}$ stain. [18,19] The said pattern of AFB positivity shows an inverse relation with the presence of epithelioid cell granulomas and a direct one with necrotic material, the reason being that liquefaction of a necrotic focus is associated with an enhanced proliferation of AFB. This pattern of $\mathrm{ZN}$ positivity was noted with the study conducted by Bibbo et al [20], Das et al [12] Gupta et al [13], Chakraborti et al [21] and Malakar et al [22]. Therefore, cases showing epithelioid cells without necrosis and a negative $\mathrm{ZN}$ stain are inconclusive cases. According to Singh et al in India the presence of epithelioid cell granulomas forms the basis of diagnosis of tuberculous lymphadenitis $[1,23,24,27]$.

The demonstration of an AFB is diagnostic of tuberculosis, therefore only $\mathrm{ZN}$ negative cases were selected for culture. Out of the $53 \mathrm{ZN}$ negative cases aspirates from 40 cases were inoculated on LJ media. The other 13 cases couldn't be inoculated on culture media because of insufficient material. Ten out of these 40 cases were positive, making the overall culture positivity $25 \%$. In the present study low culture positivity could be attributed to paucity of organisms in the lesion as $10-1000 \mathrm{bacilli} / \mathrm{mm} 3$ of the specimen is required to obtain a positive result on culture [25]. Other variable causes like the course of natural healing, history of previous anti tubercular treatment, unrepresentative lymph node aspirate and presence of 
bacteriostatic substances. The low culture positivity in the present study is in accordance with the other studies carried out $[2,8,26]$. However, Natraj et al in their study reported culture positivity between 50 to $83.3 \%$, regarding the same, culturing pus rather than the tissue may not show growth as bacilli in the pus are already killed by free fatty acids $[10,27]$ Hence resulting in low culture positivity.

Tuberculosis is recognized as the one of the most common opportunistic infection seen in HIV seropositive patients presenting as pulmonary, extra pulmonary and disseminated disease. In the current study out of 117 patients of tuberculous lymphadenitis 16 were HIV positive amongst which 12 cases had presented with regional lymphadenopathy and remaining 4 showed generalized lymphadenopathy. These findings were in contrast to the study conducted by Jayaram et al [28] who found generalized lymphadenopathy in most of the cases. Associated pulmonary tuberculosis was seen in $31 \%$ and this was in accordance with study conducted by Rajshekhran et al [9].

Cytomorphologically no particular pattern was found to be specific for tuberculous lymphadenitis in seropositive patients as reported by others [29] although necrotizing lymphadenitis has been considered to be distinctive pattern for diagnosis of tuberculosis in AIDS patients by Ltaljos et al $[31,33]$. In our study a confident diagnosis of tuberculosis could be given in 11 cases. The overall ZN positivity in seropositive patients was $68.75 \%$ as compared to $54.7 \%$ in seronegative patients. Culture positivity was also higher in seronegative patients. Similar findings were observed by Nayak et al [29], Arora et al [34], Prasoon et al [30] and Radhika et al [35].

The low rates of positivity on culture and a long wait of 8 weeks before the report can be dispatched questions the actual efficacy and utility of this procedure and hence calls for better and rapid techniques with a higher sensitivity. Flurochrome staining using auramine / rhodamine stains has a popular approach with better sensitivity index. Flurochrome stained smears may be restained with $\mathrm{ZN}$ after examination without removing auramine.

On the other perspective despite its usefulness in the diagnosis of tuberculous lymphadenitis, fine needle aspiration cytology (FNAC) faces several limitations, and its sensitivity and specificity are not well established. Advancements made in the field of molecular and immunological techniques have provided alternative approaches for rapid and reliable diagnosis. These include Polymerase Chain reaction (PCR) and DOT ELISA. In a study conducted by Aljafari et al, the diagnostic accuracy and limitations of FNAC were studied in comparison with conventional microbiological methods and polymerase chain reaction (PCR). Sixty patients with lymphadenopathy and a clinical diagnosis of tuberculous lymphadenitis were subjected to FNA. The aspirate was used for cytological examination, Ziehl-Neelsen staining, mycobacterial culture and PCR. PCR was performed using two sets of oligonucleotide primers for Mycobacterium tuberculosis and a single primer for M.Bovis species. In this study, the results of FNAC, microbiological methods and PCR correlated with the clinical outcome after follow-up for an average period of 24 months. Twentyfive cases $(41.6 \%)$ were treated and responded well to anti-tuberculosis therapy, among them 17 were correctly diagnosed by FNAC (68\%), eight by microbiological methods (32\%) and 24 by PCR $(96 \%)$.

Where PCR is considered the gold standard, FNAC predicted the correct diagnosis in $62 \%$ of cases with a high false negative rate $(38 \%)$ due to the absence of granuloma/necrosis in smears from cases of early tuberculosis. In the latter group PCR proved to be the most valuable and a diagnostic success of $100 \%$ was achieved when FNAC and PCR were combined. In addition, PCR allowed immediate characterization of M. tuberculosis in the vast majority $(96.2 \%)$ of cases in the study population [32].

In addition to being simple and inexpensive ELISA is very sensitive and rapid in diagnosing pulmonary and extra pulmonary tuberculosis. The sensitivity and specificity of ELISA has been found to be $95.4 \%$ and $88.5 \%$ respectively. In comparison to PCR, ELISA is more sensitive and less specific mainly because of its high specificity index of MOTT antigens detection PCR can demonstrate mycobacterial DNA in lymph nodes even in culture and smear negative cases. Therefore, ELISA is a rapid, easy and cost effective screening test which can be used in AFB negative cases of tuberculous lymphadenitis.

\section{Conclusion}

Tuberculous lymphadenitis was one of the most common types of lymphadenitis encountered in our outpatient department. In the present study a total of 
170 clinically suspected patients of tuberculous lymphadenitis underwent FNAC and 117 were diagnosed as tuberculous lymphadenitis based on cytomorphological features, ZN staining, culture examination and clinical follow up. The low $\mathrm{ZN}$ and culture positivity should not be considered to be a disadvantage of FNAC in diagnosing tuberculous etiology as epithelioid cell granulomas were sufficient to establish the diagnosis and can be useful as first line diagnostic tool in diagnosing tuberculous lymphadenitis coupled with $\mathrm{ZN}$ staining and culture examination. However the diagnostic accuracy of FNAC can be enhanced by combination of ELISA, PCR and molecular techniques.

Funding: Nil, Conflict of interest: None initiated, Permission from IRB: Yes

\section{References}

1. Metre MS, Jayaram G. Acid-fast bacilli in aspiration smears from tuberculous lymph nodes. An analysis of 255 cases. Acta Cytol. 1987 Jan-Feb;31(1):17-9.

2. Verma K, Kapila K. Aspiration cytology for diagnosis of tuberculosis--perspectives in India. Indian J Pediatr. 2002 Nov;69 Suppl 1:S39-43.

3. Jain A, Verma RK, Tiwari V, Goel MM. Dot-ELISA vs. PCR of fine needle aspirates of tuberculous lymphadenitis: a prospective study in India. Acta Cytol. 2005 Jan-Feb;49(1):17-21.

4. Chauhan LS. Challenges for the RNTCP in India. J Indian Med Assoc. 2003 Mar;101(3):152-3.

5. Kant L. Extra pulmonary tuberculosis: Coming out of the shadows. Indian J Tuberculosis 2004; 51: 189-90.

6. Sharma SK, Mohan A. Extrapulmonary tuberculosis. Indian J Med Res. 2004 Oct;120(4):316-53.

7. Dandapat MC, Mishra BM, Dash SP, Kar PK. Peripheral lymph node tuberculosis: a review of 80 cases. Br J Surg. 1990 Aug;77(8):911-2.

8. Dua T, Ahmad P, Vasenwala S, Beg F, Malik A. A Correlation of cytomorphology with AFB positivity by smear and culture in tuberculous lymphadenitis. Indian Journal of tuberculosis 1996; 43:81-4.

9. Rajashekeran S, Gunasekeran M, Jayakumar DD, Jeyaganesh D, Bhanumati V. Tuberculous cervical lymphadenitis in HIV positive and negative patients. Indian journal of Tuberculosis 2001; 48:201-4.
10. Natraj G, Kurup S, Pandit A, Mehta P. Correlation of Fine Needle Aspiration Cytology smears and culture in tuberculous lymphadenitis. Journal of Post Graduate Medicine 2002; 48:113-6.

11. Seth V, Donald PR. Essentials of tuberculosis in children. New Delhi, India: Jaypee Brothers Medical Publishers Ltd 1997:100-107.

12. Das DK, Pant JN, Chachra KL, Murthy NS, Satyanarayan L, Thankamma TC, Kakkar PK. Tuberculous lymphadenitis: correlation of cellular components and necrosis in lymph-node aspirate with A.F.B. positivity and bacillary count. Indian J Pathol Microbiol. 1990 Jan;33(1):1-10.

13. Gupta AK, Nayar M, Chandra M. Critical appraisal of fine needle aspiration cytology in tuberculous lymphadenitis. Acta Cytol. 1992 May-Jun;36(3):391-4.

14. Tripathy SN, Mishra N, Patel NM, Samantray DK, Das BK, Mania RN. Place of Aspiration Biopsy in the diagnosis of Lymphadenopathy. Indian Journal of Tuberculosis 1985; 32:130-4.

15. Madhusudan MTV. Tuberculous lymphadenitis in children. Indian Pediatrics 1976; XIII:533-8.

16. Bedi RS, Thind GS, Arora VK. A clinico pathological study of Superficial lymphadenopathy in Northern India. Indian Journal of Tuberculosis 1987; 34: 189-91.

17. Ahmed SS, Akhtar S, Akhtar K, Naseem S, Mansoor T, Khalil S. Incidence of Tuberculosis from Study of Fine Needle Aspiration Cytology in Lymphadenoapathy and Acid fast Staining. Indian Journal of Community Medicine 2005; 30 (2).

18. David HL. Bactteriology of Mycobacteriosis. CDC Atlanta and US PHS, HEW, Supritendent of Documents US Govt. printing Office Washington DC 1976.

19. Katoch VM. National JALMA Institute for Leprosy and other Mycobacterial Diseases (ICMR). Indian Journal Medical Research 2006; 123:735-38.

20. Das DK, Lymph nodes. In Bibbo $M$ eds Comprehensive Cytopathology, 2nd Edition Philadelphia: Saunders Company 1997:703-730.

21. Chakrabarti AK, Haider KK, Das S, Chakrabarti S. Morphological classification of tuberculous lesions: preliminary observations. Indian journal of 
Tuberculosis 1994; 41: 139-42.

22. Malakar D Jajoo ILN, Swarup K,Gupta OP,Jain AP, Poflee VW. Clinical Evaluation of Fine Needle Aspiration Cytology in the diagnosis of lymphadenopathy. Indian Journal tuberculosis 1991; 38:17-19.

23. Heerde PV, Miliakaus J. Lymph nodes. In Orell SR, Sterrett GF, Whitaker D eds. Fine Needle Aspiration Cytology. 4th edition New Delhi:Churchill Livingstone, 2005;83-124.

24. Singh UR, Bhatia A, Gadre DV, Talwar V. Cytologic diagnosis of tuberculous lymphadenitis in children by fine needle aspiration. Indian $\mathrm{J}$ Pediatric. 1992 Jan-Feb;59(1):115-8.

25. Bayazit YA, Bayazit N, Namiduru M. Mycobacterial cervical lymphadenitis. ORL J Otorhinolaryngol Relat Spec. 2004;66(5):275-80.

26. Jindal N, Devi B, Aggarwal A. Mycobacterial cervical lymphadenitis in childhood. Indian J Med Sci. 2003 Jan;57(1):12-5.

27. Verenker MP, Kamath K, Pinto WMJ, Rodrigues S, Pinto WRG. Mycobacterial study of Fine Needle Aspiration in Cervical Lymphadenitis. Indian Journal Tuberculosis 1996; 43: 187-89.

28. Jayaram G, Chew MT. Fine needle aspiration cytology of lymph nodes in HIV-infected individuals. Acta Cytol. 2000 Nov-Dec;44(6):960-6.

29. Nayak S, Puranik SC, Deshmukh SD, Mani R, Bhore AV, Bollinger RC. Fine Needle Aspiration
Cytology in Tuberculous lymphadenitis of patients with and without HIV infections. Diagnostic Cytopathol 2003; 31(4):205-206.

30. Prasoon D. Acid-fast bacilli in fine needle aspiration smears from tuberculous lymph nodes. Where to look for them. Acta Cytol. 2000 May-Jun;44(3):297-300.

31. Paliwal Nidhi, Thakur Sapna, Mullick Shalini and Gupta Kumud. FNAC in Tuberculous lymphadenitis: Experience from a Tertiary level reference centre. Indian Journal of tuberculosis Indian J Tuberc 2011; 58: 102-7.

32. Aljafari AS, Khalil EA, Elsiddig KE, El Hag IA, Ibrahim ME, Elsafi ME, Hussein AM, Elkhidir IM, Sulaiman GS, Elhassan AM. Diagnosis of tuberculous lymphadenitis by FNAC, microbiological methods and PCR: a comparative study. Cytopathology. 2004 Feb;15(1):44-8.

33. Llatjos M, Romeu J, Clottet B et al. Distinctive Cytology pattern for diagnosing tuberculous lymph node in AIDS $\mathbf{J}$ Acquired Immune Deficiency Syndrome1993;6:1335-338.

34. Arora B, Arora DR. Fine needle aspiration cytology in diagnosis of tuberculous lymphadenitis. Indian J Med Res. 1990 May;91:189-92.

35. Radhika S, Gupta SK, Chakrabarti A, Rajwanshi A, Joshi K. Role of culture for mycobacteria in fine-needle aspiration diagnosis of tuberculous lymphadenitis. Diagn Cytopathol. 1989;5(3):260-2.

\section{How to cite this article?}

Vimal S, Dharwadkar A, Chandanwale Shrish S, Verma V, Khandelwal A. Fine needle aspiration cytology in the diagnosis of Tuberculous lymphadenitis and utility of Ziehl Neelsen stain benefits and pitfalls. Int J Med Res Rev 2016;4(8):1466-1475.doi:10.17511/ijmrr.2016.i08.30. 